\title{
Generalized roll-call model for the Shapley-Shubik index
}

\author{
Sascha Kurz \\ University of Bayreuth, 95440 Bayreuth, Germany \\ sascha.kurz@uni-bayreuth.de
}

\begin{abstract}
In 1996 Dan Felsenthal and Moshé Machover considered the following model. An assembly consisting of $n$ voters exercises roll-call. All $n$ ! possible orders in which the voters may be called are assumed to be equiprobable. The votes of each voter are independent with expectation $0<p<1$ for an individual vote "yea". For a given decision rule $v$ the pivotal voter in a roll-call is the one whose vote finally decides the aggregated outcome. It turned out that the probability to be pivotal is equivalent to the Shapley-Shubik index. Here we give an easy combinatorial proof of this coincidence, further weaken the assumptions of the underlying model, and study generalizations to the case of more than two alternatives.
\end{abstract}

Keywords: simple games, influence, Shapley-Shubik index, several levels of approval $M S C: 91 \mathrm{~A} 12 ; 91 \mathrm{~A} 40,91 \mathrm{~A} 80$

\section{Introduction}

Consider a course in which there are two in-course assessments and an end-of-course examination. Assuming the 5-letter grade system from the US, what should be the course result of a student achieving a $\mathrm{D}$, a $\mathrm{C}$, and a $\mathrm{B}$ in the three assessments? In practice the most common procedure is the following: The letter grades are first converted to numerical values and a weighted mean is computed, which then is rounded and converted back to a letter grade. There may be additional rules describing the special case of a failure. Two Fs may force a final $\mathrm{F}$ or an $\mathrm{F}$ in the end-of-course examination has to result in an $\mathrm{F}$ unless it is balanced by two assessments which are graded $\mathrm{C}$ or better.

In Germany the school system, roughly spoken, splits into three different branches, where the most reputable branch is called "Gymnasium". In Bavaria there are some strict rules regulating the access to a Gymnasium. After the forth year the grads of mathematics, german, and local history and geography, ranging from 1 to 6 , are considered. Iff the sum is at most 7, then the student is allowed to access a Gymnasium without any additional acceptance test.

The underlying structure of both examples can be formalized as follows. Let $v: J^{n} \rightarrow$ $K$ be a mapping that aggregates $n$ inputs, whose values are contained in $J$, into a single output, which is contained in $K$. In our first example we have $n=3$ and $J=K=$ $\{\mathrm{A}, \mathrm{B}, \mathrm{C}, \mathrm{D}, \mathrm{F}\}$ with $\mathrm{A}>\mathrm{B}>\mathrm{C}>\mathrm{D}>\mathrm{E}$. In our second example we have $n=3$, 
$J=\{1,2,3,4,5,6\}$, and $K=\{$ denial, access $\}$ with access $>$ denial and $1>2>3>$ $4>5>6$. In the following we will assume that the sets $J$ and $K$ are strictly ordered. W.l.o.g. we will mostly use the sets $J=\{1, \ldots, j\}$ and $K=\{1, \ldots, k\}$ for two positive integers $j, k$ with the usual ordering over the integers. The school context is by far not the only area of application for these aggregation functions. Every committee that takes its decision by votes according to some specific voting rule is an example. The binary case, where $J=K=\{$ yes, no $\}$, is extensively treated in the voting literature. For examples with $(j, k) \neq(2,2)$ we refer the interested reader e.g. to [8].

A classical question in this context asks for the influence of a committee member (or voter) on the aggregated decision. To this end so-called power indices where introduced. For the binary case, the Shapley-Shubik index, introduced in [14], is one of the most commonly used power indices. Besides an axiomatic foundation of the Shapley-Shubik index [2], there is also a picturesque description: Assume that the voters express their support for a proposal one after the other. At some point the support is large enough so that the aggregated group decision will be an acceptance in any case. The corresponding voter is called a pivot. With this, the Shapley-Shubik index of a voter $i$ is the fraction of the arrangements of the voters where voter $i$ is a pivot. In [3] the model is extended to a roll-call where each voter can either say "yes" or "no". The average number of cases where a voter is pivotal coincides with the Shapley-Shubik index. Here the expectation for a "yes" need not be equal to $\frac{1}{2}$ to preserve this property. A sufficient condition is the independence and equality of expectations of the random variables for the voters, as already observed in [11] without proof. The proof in [3] uses the axiomatization of the Shapley-Shubik index as a indirect approach and reports of combinatorial difficulties for the direct approach. Here we give an easy combinatorial proof and weaken the assumptions, i.e., we assume that the probability for $s$ "yes"- and $n-s$ "no"-votes only depends on the number $s$.

The generalization of the Shapley-Shubik index to the non-binary case can be traced back at least to [4], where a third input alternative was considered. In full generality this was treated in [6], see also [16]. Here we present a consistent theory that covers the binary case, the general non-binary case, and the limiting case with an infinite number of alternatives in both the input and the output. The first basic building blocks for such a unified theory where sketched in [10].

A main assumption of our considerations is the strict ordering of the sets of alternatives. For the case of unordered alternatives we refer the interested reader to e.g. [1].

The remaining part of this paper is structures as follows. In Section 2 we define the class of games with several alternatives in the input and output. The Shapley-Shubik index for simple games is reinterpreted as a measure for uncertainty reduction in the roll-call model and generalized to the previously defined more general class of games in Section 3. The main result that the chance for being the pivotal player in a simple game is almost independent of the probability distribution of the votes is formalized in Theorem 17 in Section 4. The corresponding proof is purely combinatorial. As a possible justification of the presented generalized influence measure we present some first 
preliminary results on an axiomatization in Section 5. Games with a continuous infinite number of alternatives in the input and output are addressed in Section 6. We draw a conclusion in Section 7.

\section{Preliminaries}

Definition 1 For $J, K \subseteq \mathbb{R}$ and $n \in \mathbb{N}_{>0}$ the mapping $v: J^{n} \rightarrow K$ is called $(J, K)$ game on $n$ players. For the special sets $J=\{1, \ldots, j\}$ and $K=\{1, \ldots, k\}$, where $j=|J|$ and $k=|K|$, we speak of $(j, k)$ games and denote the set of of $(j, k)$ games on $n$ players by $\mathcal{G}_{j, k}^{n}$.

Since those mappings are not very interesting for $|K|=1$ or $|J|=1$, we assume $|J|,|K|, j, k \geq 2$ in the remaining part of the paper. We also speak of non-trivial games in order to highlight the assumption.

Definition $2 A(J, K)$ game $v$ on $n$ players is called monotonic if we have $v(a) \geq v(b)$ for all $a, b \in J^{n}$ with $a \geq b$, where $\left(a_{1}, \ldots, a_{n}\right) \geq\left(b_{1}, \ldots, b_{n}\right)$ iff $a_{i} \geq b_{i}$ for all $1 \leq i \leq$ $n$. By $\mathcal{S}_{j, k}^{n}$ we denote the set of of all monotonic $(j, k)$ games on $n$ players, which are surjective. ${ }^{1}$

We remark that $\mathcal{S}_{2,2}^{n}$ is in bijection with the set of simple games on $n$ players, see e.g. [15] for an overview on simple games. Simple games are models for simple voting situations, where each player can either vote "yes" or "no". The set $S \subseteq\{1, \ldots, n\}:=N$ of "yes"-players is called coalition. The most common formalization of a simple game is given by $\tilde{v}: 2^{N} \rightarrow\{0,1\}$ with $\tilde{v}(\emptyset)=0, \tilde{v}(N)=1$, and $\tilde{v}(S) \leq \tilde{v}(T)$ for all $\emptyset \subseteq S \subseteq$ $T \subseteq N . \tilde{v}(S)=1$ means an aggregated "yes" and $\tilde{v}(S)=0$ an aggregated "no". The set notation is just an abbreviation to state that the players in $S$ are voting "yes" (or 1) and the players in $N \backslash S$ are voting "no" (or 0 ). The bijection from $2^{N}$ to $\{0,1\}^{n}$ is just the characteristic vector of a set. It is easy to check that a simple game on $n$ players is equivalent to a $(\{0,1\},\{0,1\})$ game. In the corresponding $(2,2)$ game the inputs and outputs are just increased by 1 . We remark that in the field of threshold logic the representation of a (weighted) simple game with $J=K=\{-1,1\}$ is more common.

Definition 3 Two players $1 \leq i, h \leq n$ are called equivalent in a $(J, K)$ game $v$ on $n$ players, if we have $v(a)=v(\pi(a))$ for all $a \in J^{n}$, where $\pi$ is the transposition between $i$ and $h$.

A classical question in this context asks for the influence of a committee member (or voter) on the aggregated decision. For a simple game $\tilde{v}: 2^{N} \rightarrow\{0,1\}$ the so-called Shapley-Shubik index, see [14], of player $1 \leq i \leq n$ in $\tilde{v}$ is given by

$$
\tilde{\varphi}_{i}(\tilde{v})=\frac{1}{n !} \cdot \sum_{\emptyset \subseteq S \subseteq N \backslash\{i\}}|S| ! \cdot(n-1-|S|) ! \cdot(v(S \cup\{i\})-v(S)) .
$$

\footnotetext{
${ }^{1}$ In [6] these objects were called $(j, k)$ simple games related to the notion of simple games, where the inputs are labeled in the reverse order. However, in the older papers "simple" just refers to the binary input and output. Being more precise, some authors speak of monotonic simple games.
} 
It can be interpreted as a weighted marginal contribution and is just a specialization of the Shapley value for transferable utility (TU) games, see [13]. In the next section we will describe an influence measure for $(j, k)$ games similar to the Shapley-Shubik index.

The extreme case of having absolutely no influence is captured by:

Definition 4 Let $v$ be a $(J, K)$ game on $n$ players. If we have

$$
v\left(a_{1}, \ldots, a_{n}\right)=v\left(a_{1}, \ldots, a_{i-1}, x, a_{i+1}, \ldots, a_{n}\right)
$$

for all $a_{1}, \ldots, a_{n}, x \in J$, player $i$ is called a null player.

Of course this general definition is consistent with the definition of a null player in a simple game $\tilde{v}$ and we have $\tilde{\varphi}_{i}(\tilde{v})=0$ for each null player $i$. For equivalent players $h, i$ we have $\tilde{\varphi}_{h}(\tilde{v})=\tilde{\varphi}_{i}(\tilde{v})$.

\section{The Shapley-Shubik index as a measurement for the re- duction of uncertainty}

If the votes of all $n$ players are known, then the aggregated decision is uniquely determined by the underlying game. In order to evaluate the influence of each player on the final outcome, one can think of the voting situation as a roll-call, c.f. [3]. Each player declares her vote one after the other. In the case of a binary outcome, i.e., $|K|=2$, there exists a certain player $i$ whose declaration uniquely determines the outcome for the first time. The respective player is commonly called pivotal, cf. Definition 10. Of course the pivotality depends on the ordering of the players in this context. For more than two output alternatives the set of possible outcomes may shrink several times.

Definition 5 Let $v$ be a $(J, K)$ game on $n$ players and $S_{n}$ be the set of all bijections of $N$, i.e., the set of all permutations of $N=\{1, \ldots, n\}$. For each $1 \leq h \leq n$ we set $\tau_{h}: \mathcal{G}_{J, K}^{n} \times S_{n} \times J^{n} \rightarrow\{0, \ldots,|K|-1\}$,

$$
\begin{aligned}
\tau_{h}\left(v, \pi, a_{1}, \ldots, a_{n}\right)= & \mid\left\{v\left(a_{1}^{\prime}, \ldots, a_{n}^{\prime}\right): a_{l}^{\prime}=a_{l} \text { if } \pi(l)<h \text { and } a_{l}^{\prime} \in J \text { otherwise }\right\} \mid \\
& -\mid\left\{v\left(a_{1}^{\prime}, \ldots, a_{n}^{\prime}\right): a_{l}^{\prime}=a_{l} \text { if } \pi(l) \leq h \text { and } a_{l}^{\prime} \in J \text { otherwise }\right\} \mid,
\end{aligned}
$$

where $\mathcal{G}_{J, K}^{n}$ denotes the set of $(J, K)$ game on $n$ players.

So, given an ordering $\pi$ of the $n$ players and a specific input $\left(a_{1}, \ldots, a_{n}\right)$, the value of $\tau_{h}\left(v, \pi, a_{1}, \ldots, a_{n}\right)$ displays the decrease of our uncertainty of the final outcome after the $h$ th player, according to $\pi$, has declared her vote. The initial uncertainty is $|\operatorname{im}(v)|-1$, where

$$
\operatorname{im}(v)=\left|\left\{v\left(a_{1}, \ldots, a_{n}\right): a_{i} \in J \forall 1 \leq i \leq n\right\}\right|
$$

denotes the image of the mapping $v$, i.e., there are $|\operatorname{im}(v)|$ possible outcomes before the first player declares her vote and a unique outcome after the last player has declared her vote. By summing over all possible orderings and all possible inputs we obtain a general measurement for influence after normalization: 
Definition 6 For each positive integer $n$, each integer $1 \leq i \leq n$, and each sets $J, K \subseteq \mathbb{R}$ with $|J|,|K| \geq 2$ we set $\varphi_{i}: \mathcal{G}_{J, K}^{n} \rightarrow \mathbb{R}_{\geq 0}$ with

$$
\varphi_{i}(v)=\frac{1}{n !} \cdot \frac{1}{|J|^{n}} \cdot \frac{1}{|\operatorname{im}(v)|-1} \cdot \sum_{\left(\pi, a_{1}, \ldots, a_{n}\right) \in S_{n} \times J^{n}} \tau_{\pi^{-1}(i)}\left(v, \pi, a_{1}, \ldots, a_{n}\right)
$$

if $|\operatorname{im}(v)|>1$ and $\varphi_{i}(v)=0$ otherwise.

We remark that we have $|\operatorname{im}(v)|=|K|$ if $v$ is surjective. Via the normalization factors, the influence measure of Definition 6 obtains a nice property that is called efficiency in the context of power indices.

Lemma 7 For each $v \in \mathcal{G}_{J, K}^{n}$ with $|\operatorname{im}(v)|>1$ we have $\sum_{i=1}^{n} \varphi_{i}(v)=1$ and $\varphi_{i}(v) \in[0,1]$ for all $1 \leq i \leq n .^{2}$

Proof. For each $\pi \in S_{n}$ and each $\left(a_{1}, \ldots, a_{n}\right) \in J^{n}$ we have

$$
\sum_{i=1}^{n} \tau_{\pi^{-1}(i)}\left(v, \pi, a_{1}, \ldots, a_{n}\right)=\sum_{h=1}^{n} \tau_{h}\left(v, \pi, a_{1}, \ldots, a_{n}\right)=|\operatorname{im}(v)|-1 .
$$

Definition 8 We call $v \in \mathcal{G}_{j, k}^{n}$ output-rough if

$$
\left\{v\left(a_{1}^{\prime}, \ldots, a_{n}^{\prime}\right): a_{l}^{\prime}=a_{l} \text { if } \pi(l) \leq h \text { and } a_{l}^{\prime} \in J \text { otherwise }\right\}
$$

is an interval ${ }^{3}$ for all $\left(a_{1}, \ldots, a_{n}\right) \in J^{n}, \pi \in S_{n}, 1 \leq h \leq n$.

We remark each $(j, 2)$ game is output-rough. An example of a surjective and monotonic $(2,3)$ game that is not output-rough ins given in Example 19. An example of a surjective, monotonic, output-rough $(2,3)$ game $v$ is given by $v(1,1)=1, v(2,2)=3$, and $v(1,2)=v(2,1)=2$.

For the case of surjective, monotonic, output-rough $(j, k)$ games with $j, k \geq 2$, Equation (2) can be simplified significantly.

Lemma 9 For each non-trivial, surjective, monotonic, output-rough $(j, k)$ game $v$ on $n$ players and each integer $1 \leq i \leq n$ we have

$$
\begin{aligned}
\varphi_{i}(v)= & \frac{1}{n !} \cdot \frac{1}{j^{n}} \cdot \frac{1}{k-1} . \\
& \sum_{\left(\pi, a=\left(a_{1}, \ldots, a_{n}\right)\right) \in S_{n} \times J^{n}}\left(\bar{v}_{\pi, \pi^{-1}(i)-1}(a)-\underline{v}_{\pi, \pi^{-1}(i)-1}(a)\right)-\left(\bar{v}_{\pi, \pi^{-1}(i)}(a)-\underline{v}_{\pi, \pi^{-1}(i)}(a)\right),
\end{aligned}
$$

where $\bar{v}_{\pi, h}(a)=v\left(a_{1}^{\prime}, \ldots, a_{n}^{\prime}\right)$ with $a_{l}^{\prime}=a_{l}$ if $\pi(l) \leq h$ and $a_{l}^{\prime}=j$ otherwise; $\underline{v}_{h}(b)=$ $v\left(b_{1}^{\prime}, \ldots, b_{n}^{\prime}\right)$ with $b_{l}^{\prime}=b_{l}$ if $\pi(l) \leq h$ and $b_{l}^{\prime}=1$ otherwise.

\footnotetext{
${ }^{2}$ We remark that the Shapley-Shubik index for simple games, based on Equation (1), is not efficient in all non-monotonic cases. For $\tilde{v}(\emptyset)=v(\{2\})=v(\{1,2\})=0, \tilde{v}(\{1\})=1$ we would have $\tilde{\varphi}_{1}(\tilde{v})=\tilde{\varphi}_{2}(\tilde{v})=0$.

${ }^{3}$ We call a set $M \subseteq \mathbb{Z}$ an interval if there exist $a, b \in \mathbb{R}$ with $M=[a, b] \cap \mathbb{Z}$.
} 
Proof. Due to monotonicity and output-roughness we have

$$
\left\{v\left(a_{1}^{\prime}, \ldots, a_{n}^{\prime}\right): a_{l}^{\prime}=a_{l} \text { if } \pi(l) \leq h \text { and } a_{l}^{\prime} \in J \text { otherwise }\right\}=\left\{\underline{v}_{\pi, h}(a), \ldots, \bar{v}_{\pi, h}(a)\right\}
$$

for all $1 \leq h \leq n$ and all $a=\left(a_{1}, \ldots, a_{n}\right) \in J^{n}$.

For $j=k=2$ the formula for $\varphi_{i}$ can be simplified significantly, which is the topic of the next section. Some more notation can be introduced for the slightly more general case $k=2$.

Definition 10 For $j \geq 2$, let $v$ be a surjective $(j, 2)$ game on $n$ players. Given an input vector $a \in J^{n}$ and an ordering $\pi \in S_{n}$ we call a player $1 \leq i \leq n$ a pivot for $a, \pi$ in $v$ if $\tau_{\pi^{-1}(i)}(v, \pi, a)=1$.

In other words, a player $i$ is a pivot, if the declarations before player $i$ still allow both elements of $K$ as possible outcomes while player $i$ fixes the outcome (to either 1 or 2). For a non-trivial, surjective, monotonic $(j, k)$ game $v$ we may describe

$$
M=\left\{v\left(a_{1}^{\prime}, \ldots, a_{n}^{\prime}\right): a_{l}^{\prime}=a_{l} \text { if } \pi(l) \leq \pi(i) \text { and } a_{l}^{\prime} \in J \text { otherwise }\right\}
$$

by $b_{1}^{M}, \ldots, b_{k-1}^{M} \in\{-1,0,1\}$ with

- $b_{h}^{M}=-1$ iff $m \leq h$ for all $m \in M$;

- $b_{h}^{M}=1$ iff $m>h$ for all $m \in M$;

- $b_{h}^{M}=0$ otherwise.

If $b_{h}^{M}$ switches from a zero to a non-zero value, then we may call player $i$ an $h$-pivot for $a, \pi$ in $v$. The number of pivots for given $a, \pi$ is $k-1$. With this, $\varphi_{i}(v)$ is equal to the probability of a player $i$ to be pivotal, assuming equiprobable input vectors and orderings.

The notion of minimal winning coalitions can be generalized to non-trivial, surjective, monotonic $(j, 2)$ games.

Definition 11 Let $v$ be a non-trivial, surjective, monotonic $(j, 2)$ game on $n$ players. A vector $a \in J^{n}$ is called $a$ winning vector if $v(a)=2$ and losing vector otherwise. If $a$ is a winning vector, but all vectors $a^{\prime}<a$ are losing, then $a$ is called a minimal winning vector. Analogously a losing vector $a$ is called maximal losing vector if $a^{\prime}$ is a winning vector for all $a^{\prime}>a .^{4}$

We remark that $v$ is uniquely described by its set of minimal winning or its set of maximal losing vectors. The minimal winning vectors of Example 18 are given by $(2,3)$ and $(3,2)$. The corresponding maximal losing vectors are given by $(1,3),(3,1)$, and $(2,2)$.

\footnotetext{
${ }^{4}$ We write $a>b$ for $a \geq b$ and $a \neq b$.
} 


\section{The main result}

Now we focus on the special case $j=k=2$ and relate Equation (1) with Equation (3). To this end we have to introduce some further notions for simple games.

Definition 12 Let $\tilde{v}$ be a simple game on $n$ players. A coalition $S \subseteq N \backslash\{i\}$ is called an $i$-swing if $v(S \cup\{i\})-v(S)=1$, i.e., $v(S \cup\{i\})=1$ and $v(S)=0$.

With this we can rewrite Equation (1) to

$$
\tilde{\varphi}_{i}(\tilde{v})=\frac{1}{n !} \cdot \sum_{S \text { is an } i-\text { swing }}|S| ! \cdot(n-1-|S|) ! .
$$

Let $v \in \mathcal{S}_{2,2}^{n}$ correspond to $\tilde{v}$ and $S$ be an $i$-swing for an arbitrary but fixed player $i$. We set $a_{l}=2$ if $l \in S$ and $a_{l}=1$ otherwise for all $l \in N \backslash\{i\}$. With this we have $v\left(a_{1}, \ldots, a_{i-1}, 1, a_{i+1}, \ldots, a_{n}\right)=1$ and $v\left(a_{1}, \ldots, a_{i-1}, 2, a_{i+1}, \ldots, a_{n}\right)=2$. So, if the players in $S$ are asked first, then still both outcomes 1 and 2 are possible. If player $i$ says 2 , then the outcome is fixed to 2 due to the monotonicity of $v$. There are exactly $|S|$ ! $\cdot(n-1-|S|)$ ! orderings where the set of players before player $i$ coincides with $S$. There is another interpretation: If the players of $N \backslash(S \cup\{i\})$ are asked first, then still both outcomes 1 and 2 are possible. If player $i$ says 1 , then the outcome is fixed to 1 due to the monotonicity of $v$. From these observations we conclude:

Lemma 13 Let $\tilde{v}$ be a simple game on $n$ players and $v$ be the corresponding $(2,2)$ game. We have

$$
\begin{aligned}
\tilde{\varphi}_{i}(\tilde{v}) & =\frac{1}{n !} \cdot \sum_{S \text { is an i-swing }}|S| ! \cdot(n-1-|S|) ! \\
& =\frac{1}{n !} \cdot \sum_{(\pi, a=(2, \ldots, 2)) \in S_{n} \times J^{n}}\left(\bar{v}_{\pi, \pi^{-1}(i)-1}(a)-\underline{v}_{\pi, \pi^{-1}(i)-1}(a)\right)-\left(\bar{v}_{\pi, \pi^{-1}(i)}(a)-\underline{v}_{\pi, \pi^{-1}(i)}(a)\right) \\
& =\frac{1}{n !} \cdot \sum_{(\pi, a=(1, \ldots, 1)) \in S_{n} \times J^{n}}\left(\sum_{\pi, \pi^{-1}(i)-1}(a)-\underline{v}_{\pi, \pi^{-1}(i)-1}(a)\right)-\left(\bar{v}_{\pi, \pi^{-1}(i)}(a)-\underline{v}_{\pi, \pi^{-1}(i)}(a)\right) .
\end{aligned}
$$

So, the Shapley-Shubik index is the same as the influence measure from Definition 6 when all players say 2 ("yes") or all players say 1 ("no") in the roll-call model. Equation (1) can be seen as a computational simplification. We will see shortly that the connection between Equation (1) and the roll-call model is far more general than suggested by Lemma 13. To this end denote by $\chi_{h}(a)$ the number of $a_{i}$ which are equal to $h$.

Lemma 14 For $v \in \mathcal{S}_{2,2}^{n}$ we have

$$
\left(\bar{v}_{\pi, \pi^{-1}(i)-1}(a)-\underline{v}_{\pi, \pi^{-1}(i)-1}(a)\right)-\left(\bar{v}_{\pi, \pi^{-1}(i)}(a)-\underline{v}_{\pi, \pi^{-1}(i)}(a)\right)=1
$$

iff either

$$
\underline{v}_{\pi, \pi^{-1}(i)-1}(a)=\underline{v}_{\pi, \pi^{-1}(i)}(a)=\bar{v}_{\pi, \pi^{-1}(i)}(a)=1, \bar{v}_{\pi, \pi^{-1}(i)-1}(a)=2, a_{i}=1
$$


or

$$
\underline{v}_{\pi, \pi^{-1}(i)-1}(a)=1, \underline{v}_{\pi, \pi^{-1}(i)}(a)=\bar{v}_{\pi, \pi^{-1}(i)}(a)=\bar{v}_{\pi, \pi^{-1}(i)-1}(a)=2, a_{i}=2 .
$$

Proof. Due to monotonicity the following cases are possible:

\begin{tabular}{rrrr}
\hline$\underline{v}_{\pi, \pi^{-1}(i)-1}(a)$ & $\underline{v}_{\pi, \pi^{-1}(i)}(a)$ & $\bar{v}_{\pi, \pi^{-1}(i)}(a)$ & $\bar{v}_{\pi, \pi^{-1}(i)-1}(a)$ \\
\hline 1 & 1 & 1 & 1 \\
1 & 1 & 1 & 2 \\
1 & 1 & 2 & 2 \\
1 & 2 & 2 & 2 \\
2 & 2 & 2 & 2 \\
\hline
\end{tabular}

Lemma 15 For integers $0 \leq s \leq h-1 \leq n-1$ we have

$$
\sum_{l=0}^{n-h}\left(\begin{array}{c}
s+l \\
s
\end{array}\right) \cdot\left(\begin{array}{c}
n-s-1-l \\
h-s-1
\end{array}\right)=\left(\begin{array}{l}
n \\
h
\end{array}\right) .
$$

Proof. The stated summation formula can be concluded from Vandermonde's Identity. Here we give a direct combinatorial proof by double counting. The number of ways to choose $h$ out of $n$ objects is given by $\left(\begin{array}{l}n \\ h\end{array}\right)$. For a selection let $l$ be an integer such that the $(s+1)$ th chosen object is labeled $s+l+1$, where we assume labels from 1 to $n$. Here $l$ can range from 0 to $n-h$ and is uniquely determined. Since exactly $s$ elements have to be chosen before the $(s+1)$ th element and $h-s-1$ elements have to be chosen after the $(s+1)$ th element, there are $\left(\begin{array}{c}s+l \\ s\end{array}\right) \cdot\left(\begin{array}{c}n-s-1-l \\ h-s-1\end{array}\right)$ possibilities. Summing over the possible values for $l$ gives the stated formula.

Lemma 16 Let $\tilde{v}$ be a simple game on $n$ players and $v$ be the corresponding $(2,2)$ game. For each $1 \leq i \leq n$ and each $0 \leq h \leq n$ we have

$$
\begin{aligned}
& \left(\begin{array}{l}
n \\
h
\end{array}\right) \cdot \sum_{S \text { is an i-swing }}|S| ! \cdot(n-1-|S|) ! \\
= & \sum_{(\pi, a) \in S_{n} \times J^{n} \text { with } \chi_{2}(a)=h}\left(\bar{v}_{\pi, \pi^{-1}(i)-1}(a)-\underline{v}_{\pi, \pi^{-1}(i)-1}(a)\right)-\left(\bar{v}_{\pi, \pi^{-1}(i)}(a)-\underline{v}_{\pi, \pi^{-1}(i)}(a)\right) .
\end{aligned}
$$

Proof. We prove the stated equation by double counting. The right hand side clearly counts the number of cases $(\pi, a) \in S_{n} \times J^{n}$ with $\chi_{2}(a)=h$ where Equation (4) is satisfied.

Now let $\pi \in S_{n}$ and $a \in J^{n}$ with $\chi_{2}(a)=h$, where Equation (4) is satisfied, be arbitrary but fixed. Set $F=\{l \in N: \pi(l)<\pi(i)\}$ and $B=\{l \in N: \pi(l)>\pi(i)\}$, so that $F \cup\{i\} \cup B=N$ is a partition. If $a_{i}=2$, then we set $S=\left\{l \in F: a_{l}=2\right\}$. If $a_{i}=1$, then we choose $S \subseteq N \backslash\{i\}$ such that $\left\{l \in F: a_{l}=1\right\}=N \backslash(S \cup\{i\})$. We can easily check that $S$ is an $i$-swing in both cases.

Now we start with a fixed $i$-swing $S$ and count the corresponding pairs $(\pi, a)$ as described above. We can easily check that exactly one of the cases $|S| \leq h-1$ or $|N \backslash(S \cup\{i\})| \leq n-h-1$ is satisfied. 
- For the second case of Lemma 14 we need $|S| \leq h-1$ and $a_{m}=2$ for all $m \in S \cup\{i\}$. Exactly $h-s-1$ out of the remaining $n-s-1$ players have to vote 2 so that $\chi_{2}(a)=h$. Choose a integer $l$ so that the set $F$, as described above, has cardinality $|S|+l$. Thus, we have $|B|=n-|S|-1-l, 0 \leq l \leq n-h$ and $l$ out of the $n-h$ players voting 1 have to be chosen for $F$. Since there are $|F| ! \cdot|B|$ ! fitting permutations $\pi$, we obtain

$$
\begin{aligned}
&\left(\begin{array}{c}
n-s-1 \\
h-s-1
\end{array}\right) \cdot \sum_{l=0}^{n-h}(s+l) ! \cdot(n-s-1-l) ! \cdot\left(\begin{array}{c}
n-h \\
l
\end{array}\right) \\
&= \sum_{l=0}^{n-k} \frac{(n-s-1) ! \cdot(s+l) ! \cdot(n-s-1-l) ! \cdot(n-h) !}{(h-s-1) ! \cdot(n-h) ! \cdot l ! \cdot(n-h-l) !} \\
&= s ! \cdot(n-s-1) ! \cdot \sum_{l=0}^{n-h}\left(\begin{array}{c}
s+l \\
s
\end{array}\right) \cdot\left(\begin{array}{c}
n-s-1-l \\
h-s-1
\end{array}\right) \\
& \stackrel{\text { Lemma } 15}{=}\left(\begin{array}{c}
n \\
h
\end{array}\right) \cdot s ! \cdot(n-s-1) !
\end{aligned}
$$

cases, where we use $|S|=s$ as abbreviation.

- For the first case of Lemma 14 we need $|N \backslash(S \cup\{i\})| \leq n-h-1$ and $a_{m}=1$ for all $m \in N \backslash S$. Exactly $s-h$ out of the remaining $s$ players have to vote 1 so that $\chi_{2}(a)=h$. Choose a integer $l$ so that the set $F$, as described above, has cardinality $n-s-1+l$. Thus, we have $|B|=s-l, 0 \leq l \leq h$ and $l$ out of the $h$ players voting 2 have to be chosen for $F$. Since there are $|F|$ ! $\cdot|B|$ ! fitting permutations $\pi$, we obtain

$$
\begin{aligned}
&\left(\begin{array}{c}
s \\
s-h
\end{array}\right) \cdot \sum_{l=0}^{h}(n-s-1+l) ! \cdot(s-l) ! \cdot\left(\begin{array}{l}
h \\
l
\end{array}\right) \\
&=\quad s ! \cdot(n-s-1) ! \cdot \sum_{l=0}^{h}\left(\begin{array}{c}
n-s-1+l \\
n-s-1
\end{array}\right) \cdot\left(\begin{array}{c}
s-l \\
s-h
\end{array}\right) \\
& \stackrel{\text { Lemma } 15}{=}\left(\begin{array}{c}
n \\
n-h
\end{array}\right) \cdot s ! \cdot(n-s-1) !=\left(\begin{array}{l}
n \\
h
\end{array}\right) \cdot s ! \cdot(n-s-1) !
\end{aligned}
$$

cases.

Applying Lemma 16 yields our main result:

Theorem 17 Let $\tilde{v}$ be a simple game on $n$ players, $v$ be the corresponding $(2,2)$ game, and $p: J^{n} \rightarrow[0,1]$ a probability measure with $p(a)=p(b)$ for all $a, b \in J^{n}$ with $\chi_{2}(a)=$ $\chi_{2}(b)$. For each $1 \leq i \leq n$ we have

$$
\tilde{\varphi}_{i}(\tilde{v})=\frac{1}{n !} \cdot \sum_{\left(\pi, a=\left(a_{1}, \ldots, a_{n}\right)\right) \in S_{n} \times J^{n}} p(a) \cdot\left(\left(\bar{v}_{\pi, \pi^{-1}(i)-1}(a)-\underline{v}_{\pi, \pi^{-1}(i)-1}(a)\right)-\left(\bar{v}_{\pi, \pi^{-1}(i)}(a)-\underline{v}_{\pi, \pi^{-1}(i)}(a)\right)\right) .
$$


We remark that for $p(a)=q^{\chi_{2}(a)} \cdot(1-q)^{n-\chi_{2}(a)}$ we obtain the (unproven) result that the Shapley-Shubik index of a player $i$ in a simple game is equal to the probability of $i$ being pivotal, where the players' votes are independent and the individual "yes"-votes have an expectation of $0 \leq q \leq 1$, cf. [11].

As a refinement we weaken the assumption that the individuals votes are independent to, lets say, anonymous probabilities ${ }^{5}$ for the votes, i.e., the probability for a vector of votes only depends on the number of "yes"-votes, which seems to be a very reasonable assumption.

Setting $p(2, \ldots, 2)=1$ and $p(a)=0$ otherwise, we can obtain Equation (1) as a computational simplification of the general roll-call model with anonymous probabilities for the votes in Theorem 17. (If $a_{i}=2$ for all $1 \leq i \leq n$ we can simplify

$$
\left(\bar{v}_{\pi, \pi^{-1}(i)-1}(a)-\underline{v}_{\pi, \pi^{-1}(i)-1}(a)\right)-\left(\bar{v}_{\pi, \pi^{-1}(i)}(a)-\underline{v}_{\pi, \pi^{-1}(i)}(a)\right)
$$

to $\underline{v}_{\pi, \pi^{-1}(i)}(a)-\underline{v}_{\pi, \pi^{-1}(i)-1}(a)$, which is equivalent to $v(S \cup\{i\})-v(S)$ for $S=\{l \in N$ : $\pi(l)<\pi(i)\}$.)

For $p(a)=\frac{1}{2^{n}}$ we obtain $\tilde{\varphi}_{i}(\tilde{v})=\varphi_{i}(v)$, which gives some justification for calling the influence measure from Definition 6 the Shapley-Shubik index for $(j, k)$ games. Using the correspondence between $i$-swings and input vectors satisfying Equation (4) from the proof of Theorem 17 we can directly prove this equation. Starting from an $i$-swing $S$ we write the set $F$ as $S \cup X$ and set $|X|=x$. In contrast to the proof of Theorem 17 we make no assumption on the values of $a_{l}$ for $l \in B$. So, we have $\left(\begin{array}{c}n-s-1 \\ x\end{array}\right)$ possibilities for $X, 2^{n-s-x-1}$ possibilities for $a$, and $(s+x) ! \cdot(n-s-x-1)$ ! possibilities for $\pi$, where $0 \leq x \leq n-s-1$. We compute

$$
\begin{aligned}
& \sum_{x=0}^{n-s-1}(s+x) ! \cdot(n-s-x-1) ! \cdot\left(\begin{array}{c}
n-s-1 \\
x
\end{array}\right) \cdot 2^{n-s-x-1} \\
= & s ! \cdot(n-s-1) ! \cdot \sum_{x=0}^{n-s-1}\left(\begin{array}{c}
s+x \\
x
\end{array}\right) \cdot 2^{n-s-x-1} \\
= & s ! \cdot(n-s-1) ! \cdot \sum_{y=0}^{n-s-1}\left(\begin{array}{c}
n-1-y \\
n-s-1-y
\end{array}\right) \cdot 2^{y}
\end{aligned}
$$

corresponding possibilities.

For the first case of Lemma 14 we similarly have $\left(\begin{array}{c}s \\ x\end{array}\right)$ possibilities for $X, 2^{s-x}$ possibilities for $a$, and $(n-s-1+x) ! \cdot(s-x)$ ! possibilities for $\pi$, where $0 \leq x \leq s$. We

\footnotetext{
${ }^{5}$ Cf. [7].
} 
compute

$$
\begin{aligned}
& \sum_{x=0}^{s}(s-x) ! \cdot(n-s+x-1) ! \cdot\left(\begin{array}{l}
s \\
x
\end{array}\right) \cdot 2^{s-x} \\
= & s ! \cdot(n-s-1) ! \cdot \sum_{x=0}^{s}\left(\begin{array}{c}
n-s-1+x \\
x
\end{array}\right) \cdot 2^{s-x} \\
= & s ! \cdot(n-s-1) ! \cdot \sum_{y=0}^{s}\left(\begin{array}{c}
n-1-y \\
s-y
\end{array}\right) \cdot 2^{y}
\end{aligned}
$$

corresponding possibilities.

The equation $\tilde{\varphi}_{i}(\tilde{v})=\varphi_{i}(v)$, which was indirectly proven in [3] now follows from

$$
\left(\sum_{y=0}^{n-s-1}\left(\begin{array}{c}
n-1-y \\
n-s-1-y
\end{array}\right) \cdot 2^{y}\right)+\left(\sum_{y=0}^{s}\left(\begin{array}{c}
n-1-y \\
s-y
\end{array}\right) \cdot 2^{y}\right)=2^{n} .
$$

This is indeed a very interesting identity on its own. According to the computer algebra system Maple the first sum can be simplified to $2^{n}-\frac{1}{2} \cdot\left(\begin{array}{l}n \\ s\end{array}\right) \cdot \operatorname{hypergeom}\left([1, n+1],, \frac{1}{2}\right)$ and the second sum can be simplified to $2^{n}-\frac{1}{2} \cdot\left(\begin{array}{c}n \\ s+1\end{array}\right) \cdot \operatorname{hypergeom}\left([1, n+1],[s+2], \frac{1}{2}\right)$. In the theory of hypergeometric series, see e.g. [9], the corresponding identity

$$
\left(\begin{array}{l}
n \\
s
\end{array}\right) \cdot \text { hypergeom }\left([1, n+1], \frac{1}{2}\right)+\left(\begin{array}{c}
n \\
s+1
\end{array}\right) \cdot \operatorname{hypergeom}\left([1, n+1],[s+2], \frac{1}{2}\right)=2^{n+1}
$$

might be well known. Arguably, one can speak of "rather formidable combinatorial difficulties", as done in [3]. Plugging in small values of $s$ into Equation (5) and explicitly evaluating the second sum gives some nice explicit identities. For $s=0$ we obtain the well known geometric series $\sum_{y=0}^{n-1} 2^{y}=2^{n}-1$. For $s=1,2$ we obtain

$$
\sum_{y=0}^{n-2}(n-1-y) \cdot 2^{y}=2^{n}-n-1
$$

and

$$
\sum_{y=0}^{n-3}(n-1-y) \cdot(n-2-y) \cdot 2^{y-1}=2^{n}-\frac{n^{2}+n+2}{2} .
$$

We finish this section by two examples showing that the situation of Theorem $17 \mathrm{can}$ not be generalized to arbitrary parameters $j$ and $k$.

Example 18 Let $v$ be a $(3,2)$ game on 2 players with $v\left(a_{1}, a_{2}\right)=2$ iff $\left(a_{1}, a_{2}\right) \geq(3,2)$ or $\left(a_{1}, a_{2}\right) \geq(2,3)$. So, the game $v$ is surjective, monotonic, and output-rough. In the following table we list the pivotal player for all combinations of the input vector a and the ordering $\pi$. We assume that each player votes $l$ with probability $p_{l}$, i.e., $p_{1}, p_{2}, p_{3} \geq 0$ and $p_{1}+p_{2}+p_{3}=1$, and that the votes of the two players are independent. 


\begin{tabular}{|ccc|c|}
\hline$a \backslash \pi$ & $(1,2)$ & $(2,1)$ & $p(a)$ \\
\hline$(1,1)$ & 1 & 1 & $p_{1} \cdot p_{1}$ \\
$(1,2)$ & 1 & 1 & $p_{1} \cdot p_{2}$ \\
$(1,3)$ & 1 & 1 & $p_{1} \cdot p_{3}$ \\
$(2,1)$ & 2 & 1 & $p_{2} \cdot p_{1}$ \\
$(2,2)$ & 2 & 1 & $p_{2} \cdot p_{2}$ \\
$(2,3)$ & 2 & 1 & $p_{2} \cdot p_{3}$ \\
$(3,1)$ & 1 & 1 & $p_{3} \cdot p_{1}$ \\
$(3,2)$ & 1 & 1 & $p_{3} \cdot p_{2}$ \\
$(3,3)$ & 1 & 1 & $p_{3} \cdot p_{3}$ \\
\hline
\end{tabular}

Given the probabilities the (generalized) influence measure for player 2 is given by $\frac{p_{1}}{2}$ and the (generalized) influence measure for player 1 is given by $1-\frac{p_{1}}{2}$, i.e., the values are not independent from the probability distribution.

Example 19 Let $v$ be a $(2,3)$ game on 2 players with $v(2,2)=v(2,1)=3, v(1,2)=2$, and $v(1,1)=1$. So, the game $v$ is surjective and monotonic but not output-rough. In the following table we list for each player the reduction of uncertainty $\tau$ for all combinations of the input vector $a$ and the ordering $\pi$. We assume that each player votes $l$ with probability $p_{l}$, i.e., $p_{1}, p_{2} \geq 0$ and $p_{1}+p_{2}=1$, and that the votes of the two players are independent.

\begin{tabular}{|ccc|c|}
\hline$a \backslash \pi$ & $(1,2)$ & $(2,1)$ & $p(a)$ \\
\hline$(1,1)$ & $1: 1,2: 1$ & $1: 1,2: 1$ & $p_{1} \cdot p_{1}$ \\
$(1,2)$ & $1: 1,2: 1$ & $1: 1,2: 1$ & $p_{1} \cdot p_{2}$ \\
$(2,1)$ & $1: 2,2: 0$ & $1: 1,2: 1$ & $p_{2} \cdot p_{1}$ \\
$(2,2)$ & $1: 2,2: 0$ & $1: 1,2: 1$ & $p_{2} \cdot p_{2}$ \\
\hline
\end{tabular}

Given the probabilities the (generalized) influence measure for player 2 is given by $\frac{1+p_{1}}{4}$ and the (generalized) influence measure for player 1 is given by $\frac{3-p_{1}}{2}$, i.e., the values are not independent from the probability distribution.

Also for $(2,2)$ games the conditions of Theorem 17 can not be weakened too much.

Example 20 Let $v$ be the $(2,2)$ game on 3 players with $v\left(a_{1}, a_{2}, a_{3}\right)=2$ iff $a_{1}=1$ and $a_{2} \neq a_{3}$. So, $v$ is surjective and output-rough but not monotonic. In the following table we list the pivotal player for all combinations of the input vector a and the ordering $\pi$. We assume that each player votes $l$ with probability $p_{l}$, i.e., $p_{1}, p_{2} \geq 0$ and $p_{1}+p_{2}=1$, and that the votes of the two players are independent.

\begin{tabular}{|rcccccc|r|}
\hline$a \backslash \pi$ & $(1,2,3)$ & $(1,3,2)$ & $(2,1,3)$ & $(2,3,1)$ & $(3,1,2)$ & $(3,2,1)$ & $p(a)$ \\
\hline$(1,1,1)$ & 1 & 1 & 1 & 3 & 1 & 2 & $p_{1}^{3}$ \\
$(1,1,2)$ & 1 & 1 & 1 & 1 & 1 & 1 & $p_{1}^{2} p_{2}$ \\
$(1,2,1)$ & 1 & 1 & 1 & 1 & 1 & 1 & $p_{1}^{2} p_{2}$ \\
$(1,2,2)$ & 1 & 1 & 1 & 3 & 1 & 2 & $p_{1} p_{2}^{2}$ \\
$(2,1,1)$ & 3 & 2 & 3 & 3 & 2 & 2 & $p_{1}^{2} p_{2}$ \\
$(2,1,2)$ & 3 & 2 & 3 & 1 & 2 & 1 & $p_{1} p_{2}^{2}$ \\
$(2,2,1)$ & 3 & 2 & 3 & 1 & 2 & 1 & $p_{1} p_{2}^{2}$ \\
$(2,2,2)$ & 3 & 2 & 3 & 3 & 2 & 2 & $p_{2}^{3}$ \\
\hline
\end{tabular}


Given the probabilities the (generalized) influence measure for players 2 and 3 are given by $\frac{1+2 p_{2}^{2}}{6}$ and the (generalized) influence measure for player 1 is given by $\frac{4-4 p_{2}^{2}}{6}$, i.e., the values are not independent from the probability distribution.

\section{The axiomatic approach}

The meaningfulness of influence measures or power indices is commonly justified by providing some axioms which are satisfied by the measure and uniquely determine it. Here we go along these lines for our influence measure from Definition 6. To this end let $v$ be a non-trivial $(J, K)$ game on $n$ players with $|\operatorname{im}(v)|>1$. Due to Lemma 7 the influence measure $\varphi$ is efficient for $v$. For any null player $i$ in $v$ we obviously have $\varphi_{i}(v)=0$, i.e., $\varphi$ satisfies the null player axiom. For each $\pi \in S_{n}$ we define the game $\pi v$ by $(\pi v)(a)=v\left(a_{\pi(1)}, \ldots, a_{\pi(n)}\right)$. With this, we have $\varphi_{i}(\pi v)=\varphi_{\pi(i)}(v)$, which is called the anonymity axiom. The transfer axiom is satisfied if we have

$$
\varphi_{i}(u)+\varphi_{i}(w)=\varphi_{i}(u \vee w)+\varphi_{i}(u \wedge w)
$$

for all $1 \leq i \leq n$, where $(u \vee w)(a):=\max \{u(a), w(a)$ and $(u \wedge w)(a):=\min \{u(a), w(a)$.

Lemma 21 Let $u, w$ be nontrivial, surjective, monotonic, output-rough $(J, K)$ games. Then, both $u \vee w$ and $u \wedge w$ are nontrivial, surjective, monotonic, output-rough $(J, K)$ games.

Proof. Obviously $u \vee w$ and $u \wedge w$ are nontrivial, surjective, monotonic $(J, K)$ games.

Next we prove that $u \wedge w$ is output-rough. To this end let $a=\left(a_{1}, \ldots, a_{n}\right) \in J^{n}$, $\pi \in S_{n}$, and $1 \leq h \leq n$ arbitrary but fix. Choose integers $\alpha_{1}, \alpha_{2}, \beta_{1}, \beta_{2}$ such that

$$
\left\{u\left(a_{1}^{\prime}, \ldots, a_{n}^{\prime}\right): a_{l}^{\prime}=a_{l} \text { if } \pi(l) \leq h \text { and } a_{l}^{\prime} \in J \text { otherwise }\right\}=\left[\alpha_{1}, \beta_{1}\right] \cap \mathbb{Z}
$$

and

$$
\left\{w\left(a_{1}^{\prime}, \ldots, a_{n}^{\prime}\right): a_{l}^{\prime}=a_{l} \text { if } \pi(l) \leq h \text { and } a_{l}^{\prime} \in J \text { otherwise }\right\}=\left[\alpha_{2}, \beta_{2}\right] \cap \mathbb{Z} .
$$

We will prove

$$
\left\{(u \wedge w)\left(a_{1}^{\prime}, \ldots, a_{n}^{\prime}\right): a_{l}^{\prime}=a_{l} \text { if } \pi(l) \leq h \text { and } a_{l}^{\prime} \in J \text { otherwise }\right\}=\left[\alpha_{3}, \beta_{3}\right] \cap \mathbb{Z},
$$

where $\alpha_{3}=\min \left\{\alpha_{1}, \alpha_{2}\right\}$ and $\beta_{3}=\min \left\{\beta_{1}, \beta_{2}\right\}$, in the following. We set

$$
R=\left\{\left(a_{1}^{\prime}, \ldots, a_{n}^{\prime}\right) \in J^{n}: a_{l}^{\prime}=a_{l} \text { if } \pi(l) \leq h\right\} .
$$

Now let $\gamma \in\left[\alpha_{3}, \beta_{3}\right]$. If $\gamma<\alpha_{2}$ we choose a vector $r \in R$ with $u(r)=\gamma$. Since $w(r)>\gamma$, we have $(u \wedge w)(r)=\gamma$. Similarly, we can conclude the existence of an input vector $r \in R$ with $(u \wedge w)(r)=\gamma$ if $\gamma\left\langle\alpha_{1}, \gamma>\beta_{1}\right.$, or $\gamma>\beta_{2}$. In the remaining cases we have $\alpha_{1} \leq \gamma \leq \beta_{1}$ and $\alpha_{2} \leq \gamma \leq \beta_{2}$. Now let $R_{\gamma}$ be the set of elements $r^{\prime} \in R$ with $u\left(r^{\prime}\right)=\gamma$. If there exists an element $r \in R_{\gamma}$ with $w(r) \geq \gamma$, then $(u \wedge w)(r)=\gamma$. So, we 
assume $w\left(r^{\prime}\right)<\gamma$ for all $r^{\prime} \in R_{\gamma}$ and choose an arbitrary $r^{h} \in R_{\gamma}$. For each $h<l \leq n$ we define $r^{l}$ by $r_{i}^{l}=r_{i}^{l-1}$ if $\pi(i) !=l$ and $r_{i}^{l}=j$ for $\pi(i)=l$. By construction we have $r^{h}<\cdots<r^{n}=(j, \ldots, j)$ and $w\left(r^{n}\right) \geq \gamma$. Let $g$ be the smallest index with $w\left(r^{g}\right) \geq \gamma$ and $w\left(r^{g-1}\right)<\gamma$. Since $w$ is output-rough we can modify the input of player $i$ with $\pi(i)=g$ in $r^{g-1}$ to a vector $r$ such that $w(r)=\gamma$ and $r \geq r^{g-1}$. Since $u(r) \geq u\left(r^{g-1}\right) \geq \gamma$ we have $(u \wedge w)(r)=\gamma$.

For $u \vee w$ we can similarly conclude

$$
\left\{(u \vee w)\left(a_{1}^{\prime}, \ldots, a_{n}^{\prime}\right): a_{l}^{\prime}=a_{l} \text { if } \pi(l) \leq h \text { and } a_{l}^{\prime} \in J \text { otherwise }\right\}=\left[\alpha_{4}, \beta_{4}\right] \cap \mathbb{Z},
$$

where $\alpha_{4}=\max \left\{\alpha_{1}, \alpha_{2}\right\}, \beta_{4}=\max \left\{\beta_{1}, \beta_{2}\right\}$, and $\alpha_{1}, \alpha_{2}, \beta_{1}, \beta_{2}$ are chosen as above.

From Lemma 9 we conclude:

Lemma 22 For each non-trivial, surjective, monotonic, output-rough $(J, K)$ games $u$ and $w$ on $n$ players we have $\varphi_{i}(u)+\varphi_{i}(w)=\varphi_{i}(u \vee w)+\varphi_{i}(u \wedge w)$ for all $1 \leq i \leq n$.

In other words, the influence measure $\varphi$ from Definition 6 satisfies the four classical axioms, used for the first axiomatization of the Shapley-Shubik index, see [2], on the class of non-trivial, surjective, monotonic, output-rough $(J, K)$ games.

Lemma 23 Let $\gamma$ be a mapping from the set of non-trivial, surjective, monotonic $(j, 2)$ games on $n$ players to $\Delta_{n}:=\left\{\left(x_{1}, \ldots, x_{n}\right) \in[0,1]^{n}: \sum_{i=1}^{n} x_{i}=1\right\}$. If $\gamma$ satisfies the transfer axiom, then $\gamma(v)$ can be recursively computed from the values of $\gamma$ for games with a unique minimal winning coalition.

Proof. We prove by induction on the number of minimal winning coalitions. By $u_{a}$ we denote the game with unique minimal winning $a$. If the (pairwise different) minimal winning vectors of $v$ are given by $a^{1}, \ldots, a^{l}$, then we can write $v=x \vee y$, where $x=$ $u_{a^{1}} \vee \ldots u_{a^{l-1}}$ and $y=u_{a^{l}}$, so that $x$ has $l-1$ and $y$ has 1 minimal winning vector. Since $x \wedge y$ has at most $l-1$ winning vectors, we can compute recursively compute $\gamma(v)=\gamma(x)+\gamma(y)-\gamma(x \wedge y)$.

For non-trivial, surjective, monotonic $(2,2)$ games anonymity, efficiency, and the null player axiom uniquely determine the value of $\gamma$ on each game consisting of a single minimal winning vector. Here the null players obtain $\gamma_{i}=0$ and the non-null players obtain one divided by the number of non-null players. For $(j, 2)$ games with $j>2$ the situation is more involved for our influence measure from Definition 6:

Example 24 Let $v$ the the surjective, monotonic $(4,2)$ game on 3 players with unique minimal winning vector $(2,3,4)$. Next we determine the number of cases $(a, \pi)$, where each player is pivotal. For player 1 we consider the cases:

- $\pi=(1, \star, \star): a_{1}=1 \rightarrow 2 \cdot 1 \cdot 4 \cdot 4=32$ cases;

- $\pi=(2,1,3): a_{1}=1, a_{2} \in\{3,4\} \rightarrow 1 \cdot 1 \cdot 2 \cdot 4=8$ cases; 
- $\pi=(3,1,2): a_{1}=1, a_{3}=4 \rightarrow 1 \cdot 1 \cdot 4 \cdot 1=4$ cases;

- $\pi=(\star, \star, 1): a_{2} \in\{3,4\}, a_{3}=4 \rightarrow 2 \cdot 4 \cdot 2 \cdot 1=16$ cases

For player 2 we consider the cases:

- $\pi=(2, \star, \star): a_{2} \in\{1,2\} \rightarrow 2 \cdot 4 \cdot 2 \cdot 4=64$ cases;

- $\pi=(1,2,3): a_{1}=1, a_{1} \in\{2,3,4\}, a_{2} \in\{1,2\} \rightarrow 1 \cdot 3 \cdot 2 \cdot 4=24$ cases;

- $\pi=(3,2,1): a_{1}=1, a_{2} \in\{1,2\}, a_{3}=4 \rightarrow 1 \cdot 4 \cdot 2 \cdot 1=8$ cases;

- $\pi=(\star, \star, 2): a_{1} \in\{2,3,4\}, a_{3}=4 \rightarrow 2 \cdot 3 \cdot 4 \cdot 1=24$ cases.

For player 3 we consider the cases:

- $\pi=(3, \star, \star): a_{3} \in\{1,2,4\} \rightarrow 2 \cdot 4 \cdot 4 \cdot 3=96$ cases;

- $\pi=(1,3,2): a_{1}=1, a_{1} \in\{2,3,4\}, a_{3} \in\{1,2,3\} \rightarrow 1 \cdot 3 \cdot 4 \cdot 3=36$ cases;

- $\pi=(2,3,1): a_{1}=1, a_{2} \in\{3,4\}, a_{3} \in\{1,2,3\} \rightarrow 1 \cdot 4 \cdot 2 \cdot 3=24$ cases;

- $\pi=(\star, \star, 3): a_{1} \in\{2,3,4\}, a_{2} \in\{3,4\} \rightarrow 2 \cdot 3 \cdot 2 \cdot 4=48$ cases.

Thus, we have $\varphi_{1}(v)=\frac{60}{386}=\frac{5}{32}, \varphi_{2}(v)=\frac{120}{386}=\frac{10}{32}=\frac{5}{16}$, and $\varphi_{3}(v)=\frac{204}{386}=\frac{17}{32}$.

An additional property of our influence measure $\varphi$ is that for a game $v^{\prime}$ arising from $v$ by adding a null player $l$, we have $\varphi_{i}\left(v^{\prime}\right)=\varphi_{i}(v)$ for all $i \neq l\left(\right.$ and $\left.\varphi_{l}\left(v^{\prime}\right)=0\right)$.

\section{The limiting case}

By the following normalization trick we can remove the $\frac{1}{k-1}$ factor in Equation (3) in Lemma 9. Instead of $K=\{1, \ldots, k\}$ we use $K=\left\{\frac{0}{k-1}, \frac{1}{k-1}, \ldots, \frac{k-1}{k-1}\right\} \subseteq[0,1]$. Then the $\underline{v}$ - and $\bar{v}$-values contain the necessary factor itself. ${ }^{6}$ Without any substantial effect we may also relabel the set of inputs from $J=\{1, \ldots, j\}$ to $J=\left\{\frac{0}{j-1}, \frac{1}{j-1}, \ldots, \frac{j-1}{j-1}\right\} \subseteq[0,1]$. However, by simultaneously increasing $j$ and $k$ (possibly at different velocities) we obtain an approximation of a voting scheme $[0,1]^{n} \rightarrow[0,1]$. We now introduce those objects directly.

Definition $25 A([0,1],[0,1])$ game on $n$ players is a mapping $v:[0,1]^{n} \rightarrow[0,1]$. We call $v$ surjective, monotonic, or continuous if the mapping is surjective, weakly monotonic increasing, or continuous, respectively.

\footnotetext{
${ }^{6}$ By choosing another segmentation one can implement the evaluation function proposed in [6] to assign different weights to the output values.
} 
As an abbreviation, we speak of a continuous game $v$ if $v$ is a surjective, monotonic, continuous $([0,1],[0,1])$ game. Similarly to Definition 4 we call a player $i$ null player in $v$, if $v\left(x_{1}, \ldots, x_{n}\right)=v\left(x_{1}, \ldots, x_{i-1}, x_{i}^{\prime}, x_{i+1}, \ldots, x_{n}\right)$ for all $x_{1}, \ldots, x_{n}, x_{i}^{\prime} \in[0,1]$.

As the continuity in a continuous game plays the role of output-roughness in a $(j, k)$ game, we reformulate Lemma 9 to:

Definition 26 For each continuous game $v$ on $n$ players we define $\varphi_{i}(v)$ by

$\frac{1}{n !} \sum_{\pi \in S_{n}} \int_{0}^{1} \ldots \int_{0}^{1}\left(\bar{v}_{\pi, \pi^{-1}(i)-1}(a)-\underline{v}_{\pi, \pi^{-1}(i)-1}(a)\right)-\left(\bar{v}_{\pi, \pi^{-1}(i)}(a)-\underline{v}_{\pi, \pi^{-1}(i)}(a)\right) \mathrm{d} x_{1} \ldots \mathrm{d} x_{n}$, where $\bar{v}_{\pi, h}(a)=v\left(a_{1}^{\prime}, \ldots, a_{n}^{\prime}\right)$ with $a_{l}^{\prime}=a_{l}$ if $\pi(l) \leq h$ and $a_{l}^{\prime}=1$ otherwise; $\underline{v}_{h}(b)=$ $v\left(b_{1}^{\prime}, \ldots, b_{n}^{\prime}\right)$ with $b_{l}^{\prime}=b_{l}$ if $\pi(l) \leq h$ and $b_{l}^{\prime}=0$ otherwise, for all $1 \leq i \leq n$.

We remark that the influence measure from Definition 26 satisfies efficiency, anonymity, the null player and the transfer axiom. Given a continuous game $v$ one can construct a series of monotonic $(j, j)$ games $v^{j}$, where $j \rightarrow \infty$, such that the values of $\varphi_{i}\left(v^{j}\right)$ tend to $\varphi_{i}(v)$ for all $1 \leq i \leq n$.

For the examples $\hat{v}\left(x_{1}, x_{2}, x_{3}\right)=\frac{1 x_{1}^{2}+2 x_{2}^{2}+3 x_{3}^{2}}{6}$ and $\tilde{v}\left(x_{1}, x_{2}, x_{3}\right)=x_{1} x_{2}^{2} x_{3}^{3}$ the function $\varphi$ was evaluated in [10]:

$$
\varphi(\hat{v})=\left(\frac{1}{6}, \frac{2}{6}, \frac{3}{6}\right)=(0.1 \overline{6}, 0 . \overline{3}, 0.5)
$$

and

$$
\varphi(\tilde{v})=\left(\frac{35}{144}, \frac{50}{144}, \frac{59}{144}\right)=(0.2430 \overline{5}, 0.347 \overline{2}, 0.4097 \overline{2}) .
$$

While typically the evaluation of $\varphi$ for a continuous game is based on rather tedious case distinctions, there are simple formulas for special cases.

Theorem 27 Let $w_{1}, \ldots, w_{n} \in[0,1]$ with $\sum_{i=1}^{n} w_{i}=1$ and $f_{i}:[0,1] \rightarrow[0,1]$ continuous monotonic functions with $f_{i}(0)=0$ and $f_{i}(1)=1$ for all $1 \leq i \leq n$. Then, $v(x)=$ $\sum_{i=1}^{n} w_{i} \cdot f_{i}\left(x_{i}\right)$ defines a continuous game and we have $\varphi_{i}(v)=w_{i}$ for all $1 \leq i \leq n$.

Proof. Let $q \leq i \leq n$ and $\pi \in S_{n}$ be arbitrary but fixed. With $S=\{l \in N: \pi(l)<$ $\pi(i)\}$ we have

$$
\begin{aligned}
\bar{v}_{\pi, \pi^{-1}(i)-1}(x) & =\sum_{l \in S} w_{l} \cdot f_{l}\left(x_{l}\right)+\sum_{l \in N \backslash S} w_{l} \cdot f_{l}(1)=\sum_{l \in S} w_{l} \cdot f_{l}\left(x_{l}\right)+1-w(S) \\
\bar{v}_{\pi, \pi^{-1}(i)}(x) & =\sum_{l \in S} w_{l} \cdot f_{l}\left(x_{l}\right)+w_{i} \cdot f_{i}\left(x_{i}\right)+1-w(S)-w_{i} \\
\underline{v}_{\pi, \pi^{-1}(i)-1}(x) & =\sum_{l \in S} w_{l} \cdot f_{l}\left(x_{l}\right)+\sum_{l \in N \backslash S} w_{l} \cdot f_{l}(0)=\sum_{l \in S} w_{l} \cdot f_{l}\left(x_{l}\right) \\
\underline{v}_{\pi, \pi^{-1}(i)}(x) & =\sum_{l \in S} w_{l} \cdot f_{l}\left(x_{l}\right)+w_{i} \cdot f_{i}\left(x_{i}\right)
\end{aligned}
$$

so that $\left(\bar{v}_{\pi, \pi^{-1}(i)-1}(a)-\underline{v}_{\pi, \pi^{-1}(i)-1}(a)\right)-\left(\bar{v}_{\pi, \pi^{-1}(i)}(a)-\underline{v}_{\pi, \pi^{-1}(i)}(a)\right)=w_{i}$. 
So the influence distribution for the example $\hat{v}$ is no surprise, while for continuous games similar to $\tilde{v}$ no general and easy to evaluate formula is known.

The analogy to different probabilities for the discrete set of input states is a density function in the continuous case. An example with density functions $f_{1}(x)=\frac{3}{4} \cdot\left(1-x^{2}\right)$, $f_{2}(x)=f_{2}(x)=\frac{3}{8} \cdot\left(1+x^{2}\right)$ and $v(x)$ equal to the median of $x_{1}, x_{2}, x_{3}$ was computed in [10]: $\varphi_{1}(v)=\frac{554}{13440} \approx 0.04122$ and $\varphi_{2}(v)=\varphi_{3}(v)=\frac{563}{13440} \approx 0.04189$.

\section{Conclusion}

We have studied the classical Shapley-Shubik index for simple games in the roll-call model from [3] and gave a direct combinatorial proof for the fact that the expected number of cases where a certain player is pivotal is independent from the specific distribution of the "yes"- and "no"-votes, as long as the probability does only depended on the number of "yes"-votes. This generalizes the result from [3] and gives a nice and vivid description for the Shapley-Shubik index that is less artificial than previous ones. We have applied the roll-call model for the generalized case of $j \geq 2$ ordered input and $k \geq 2$ ordered output states. Some notation from simple games can be generalized in a consistent way. By considering the reduction of uncertainty we have tried to provide a more persuasive basis for the generalized Shapley-Shubik index from [6] and other places. We do not claim that this influence measure is the correct generalization of the Shapley-Shubik index and we agree with the authors of [5] that the status of the Shapley-Shubik index for ternary and more general games requires further study. For further generalizations of the ShapleyShubik index we refer the interested reader to [12]. A first set of results with respect to the axiomatic approach is presented in order justify the proposed influence measure by another approach. In any case we find it advantageous to have an influence measure at hand that can, to some extend, be consistently defined for larger classes of games with several levels of approval in the input and output. Even continuous input and output spaces make sense in decisions on e.g. rate of taxes or other continuous variables.

\section{References}

[1] E.M. Bolger. A value for games with $n$ players and $r$ alternatives. International Journal of Game Theory, 22(4):319-334, 1993.

[2] P. Dubey. On the uniqueness of the Shapley value. International Journal of Game Theory, 4(3):131-139, 1975.

[3] D.S. Felsenthal and M. Machover. Alternative forms of the Shapley value and the Shapley-Shubik index. Public Choice, 87(3-4):315-318, 1996.

[4] D.S. Felsenthal and M. Machover. Ternary voting games. International Journal of Game Theory, 26(3):335-351, 1997. 
[5] D.S. Felsenthal and M. Machover. Models and reality: the curious case of the absent abstention. In Power, Voting, and Voting Power: 30 Years After, pages 73-86. Springer, 2013.

[6] J. Freixas. The Shapley-Shubik power index for games with several levels of approval in the input and output. Decision Support Systems, 39(2):185-195, 2005.

[7] J. Freixas. Probabilistic power indices for voting rules with abstention. Mathematical Social Sciences, 64(1):89-99, 2012.

[8] J. Freixas and W.S. Zwicker. Anonymous yes-no voting with abstention and multiple levels of approval. Games and Economic Behavior, 67(2):428-444, 2009.

[9] G. Gasper and M. Rahman. Basic hypergeometric series, volume 96. Cambridge university press, 2004.

[10] S. Kurz. Measuring voting power in convex policy spaces. Economies, 2(1):45-77, 2014.

[11] I. Mann and L.S. Shapley. The a priori voting strength of the electoral college, 1964.

[12] A.E. Roth. The Shapley value: essays in honor of Lloyd S. Shapley. Cambridge University Press, 1988.

[13] L.S. Shapley. A value for $n$-person games. In H.W. Kuhn and A.W. Tucker, editors, Annals of mathematics studies (contributions to the theory of games), volume 28, pages 307-317. Princeton Univ. Press, 1953.

[14] L.S. Shapley and M. Shubik. A method for evaluating the distribution of power in a committee system. American Political Science Review, 48(03):787-792, 1954.

[15] A.D. Taylor and W.S. Zwicker. Simple games. Desirability relations, trading, pseudoweightings. Princeton, NJ: Princeton University Press. 246 p., 1999.

[16] B. Tchantcho, L.D. Lambo, R. Pongou, and B.M. Engoulou. Voters' power in voting games with abstention: Influence relation and ordinal equivalence of power theories. Games and Economic Behavior, 64(1):335-350, 2008. 